\title{
Ante la ley y la experiencia del sentido en F. Kafka'
}

\author{
"Before the Law" and the Experience of the Meaning in F. Kafka
}

\author{
SANTIAGO MARTÍN ARNEDO \\ Universidad de Granada \\ España \\ smarnedo@hotmail.com
}

(Recibido: $16-04-2020$; aceptado: 2I-II-2O2O)

Resumen. Me propongo en este artículo analizar la estructura y el sentido del relato breve Ante la ley de F.Kafka. No solo el autor lo consideró especialmente relevante, a juzgar por las veces que lo publicó,sino que condensa ejemplarmente su estrategia narrativa, pues muchos de sus rasgos se reflejan de nuevo por extenso en la novela donde se inscribe, El proceso. Las circunstancias de su gestación y la radicalidad de su planteamiento lo sitúan en una tesitura de carácter teológico, frente a otros enfoques interpretativos de perfil más sociológico o antropológico. Esta hipótesis queda reforzada cuando surgen sin dificultad paralelismos con otros relatos bíblicos. Además, las propias opiniones del autor, en quien vida y literatura aparecen tan inexorablemente unidas, apuntan más bien a una problematización de la existencia en su conjunto, y no concretamente a alguno de sus aspectos, de ahí la angustia y la incertidumbre que envuelve tanto al protagonista de sus escritos como al lector, que en vano desea encontrar una última pieza que dé sentido al conjunto.

Palabras clave: Kafka; ley; judaísmo; proceso; teología.
Abstract.This article analyses the structure and the meaning of the short story "Before the Law" by F. Kafka. It was not only very important for him, judging by how many times he published it, but it also condenses his narrative strategy: many of its features are reflected widely in the novel where it is inscribed, The Trial. The circumstances of its gestation and the radical nature of his approach give it a theological character, compared to other more sociological or anthropological approaches. This hypothesis is reinforced when parallels with other biblical stories are easily found. Furthermore, the author's own opinions, in whom life and literature appear so inexorably linked, point rather to a problematization of existence as a whole, and not specifically to any of its aspects, hence the anguish and uncertainty that surrounds both the protagonist of his writings as well as the reader, who in vain wants to find a last piece that gives meaning to the whole.

Keywords: Kafka; law; judaism; trial; theology.

\footnotetext{
${ }^{\text {I }}$ Para citar este artículo: Martín Arnedo, Santiago (202I). Ante la ley y la experiencia del sentido en F. Kafka. Álabe 23 . [www. revistaalabe.com]

DOI: IO.I5645/Alabe2O2I.23.4
} 


\section{Un relato acabado dentro de una novela inacabada.}

Como es bien sabido, la novela inconclusa El Proceso (Der Prozess), aunque empezada en el verano de I9I4, fue póstumamente publicada por Max Brod en i925, un año después de la muerte de Franz Kafka. La labor de reconstrucción del manuscrito no fue fácil para quien había recibido como última voluntad del -ahora- famoso autor la instrucción de quemar todos sus manuscritos. Todo lo que rodea a esta obra es confuso (su significado, su gestación, su composición, etc.), y la niebla que la envuelve no parece disiparse pese a la miríada de publicaciones que se han centrado sobre ella. Y es así por varias razones: unas de orden práctico y otras de orden hermenéutico. En cuanto a las de primer orden (de las del segundo nos ocuparemos a partir del segundo punto), hay que recordar que aquello con que contaba Brod cuando se propuso hacer llegar al gran público la novela de su amigo, consistía en un fajo de i6r hojas escritas a doble cara (Stach, 2OIO: 538), arrancadas de diferentes cuadernos. No había indicaciones orientativas de orden de aparición, ni de qué textos se daban por concluidos, ni sobre qué conformaba finalmente el cuerpo de los capítulos. Brod sin embargo no se dejó arredrar ante este reto y se enfrentó intrépida y pragmáticamente a la tarea de rehacer el puzle. No dudó en completar, desechar, refundir, aportar y ordenar textos, legando a la posteridad un escrito cerrado que no había encontrado nunca un remate en la cabeza de su creador, quien, desesperado, dio por buena su incompletitud (Stach, 20IO: 538).

Cierto es que Brod contaba con algunas pesquisas en su labor compilatoria (Kafka, I998: 287). Por ejemplo respecto al título, aunque no aparecía en el manuscrito original, fue elegido a raíz de una conversación personal con el autor. Brod habría escuchado además de labios del amigo la lectura de la obra, de modo que contó en gran medida con el sostén de su memoria. Toda esta información preliminar es importante tenerla en cuenta, porque, como hemos señalado, las opacidades hermenéuticas se fundamentan en razones de no solo de contenido, sino también de forma.

A esto hay que sumar la personalidad de Kafka, en quien se juntaban una voluntad de perfeccionismo desmedida (Kafka, 20IO: 278) con la incapacidad para mantener durante mucho tiempo el aliento creativo de una misma obra (279). El resultado solamente podía ser: bien un texto breve (como la mayoría de su creaciones: relatos, apuntes de diario, etc.), bien un texto largo pero incompleto, como es el caso de sus grandes novelas póstumas.

No obstante, nos encontramos dentro de la novela Elproceso con una excepción dentro de este paisaje fragmentario e inconcluso, a saber: el breve texto Ante la ley (Vor dem Gesetz), que aparece en la segunda parte del capítulo titulado En la catedral (Im Dom) (Kafka, I998: 26I-263). Fue dado por "redondeado" por su autor, de forma excepcional, teniendo en cuenta que por dicha obra avanza con pies de plomo: valga como ejemplo cuando, refiriéndose a El proceso, escribe el 29/8/I9I4 en sus diarios: "final de capítulo fallido, y es difícil que pueda continuar con acierto otro capítulo empezado” (Kafka, I995: 273). Además Ante la ley fue publicado en vida nada menos que en dos 
ocasiones, otra excepcionalidad para alguien tan poco dado a publicar: por primera vez en I9I5, en la revista independiente judía Selbstwehr y por segunda vez en I920, dentro de la antología de relatos intitulada Un médico rural (Ein Landarzt), cuando el escritor había abandonado ya a esas alturas la expectativa de acabar su novela El proceso, que había comenzado seis años antes. De todo esto no es difícil colegir que el autor debió atribuirle a este breve relato un gran valor

\section{2. ¿Inspiración teológica?}

Hay una segunda característica más, además de representar la completitud dentro de lo incompleto: el contexto biográfico de su confección. Es significativo el hecho de que Ante la ley apareciera por primera vez en una revista de carácter sionista. Dicha publicación estaba emparentada además con la asociación hebrea Bar $K o c h b a$, frecuentada por Kafka a partir de i9II. Son fechas en las que Kafka se interesa activamente por el judaísmo (Kafka, 20Io: 46). En I9Io una compañía de teatro yiddish proveniente de Lemberg había visitado Praga, y Kafka había quedado impresionado por la carga de autenticidad en sus representaciones. También se despertó su interés por el yiddish, idioma de la comunidad judía centroeuropea que utiliza caracteres hebreos, si bien haciendo uso parcialmente de la gramática y del vocabulario alemán. Lee además $L a$ historia de losjudios de Heinrich Graetz y entabla amistad con Martin Buber (I878-1965). A partir de I9I3 comenzará a estudiar hebreo. Su dedicación al sionismo fue pues cosecha tardía. De hecho, en la Carta al padre (I955: 30), reprochaba a su progenitor haberle legado una interpretación excesivamente superficial de la cultura judía.

Es verdad que la relación de Kafka con la religiosidad fue cuando menos ambigua. Los propios estudiosos de su obra se dividen entre aquellos que utilizan el judaísmo (teniendo en cuenta además sus múltiples acepciones) como clave interpretativay aquellos otros que lo infravaloran o directamente lo niegan (Grözinger, 2OI4: 26). Creo que esta relación no se puede infravalorar. Ciertamente Kafka no fue un sionista, tampoco un revolucionario. Como indica Löwy (2019: 79): "su particularidad consiste en el carácter estrictamente negativo que adquieren en su obra tanto el mesianismo judío como la utopía libertaria”. Es decir, lainfluencia de ambos se deja sentir indirectamente, simbólicamente. Sus personajes se rebelan no positiva, no activa, sino elusiva e infructuosamente. Y sin embargo, el escritor no renuncia a la denuncia de la futilidad del esfuerzo frente a un contexto opacamente perverso. La ausencia de la justicia en sus historias es ya una forma de denunciar la injusticia. Es posible señalar algo y hacerlo presente indicando su ausencia. Löwy expresa, a mi juicio, la descripción más pregnante del sentir kafkiano al señalar que su escritura expresa un "entrelazamiento sutil entre la esperanza y la desesperación” (2019: 8I). Cierto que, por un lado, no llega respuesta alguna desde los cielos (o desde la Ley), mas el acusado (el hombre) no renuncia nunca a su escucha. Sin defender el dogma religioso, tampoco se lo desacredita, de ahí su ambigüedad. Y sin embargo, creo que con 
su narración, Kafka apunta a la existencia en su conjunto, no a tal o cual aspecto, es decir, apunta a la posibilidad de un sentido general.

En este sentido, G. Scholem (I975: 2I2) aconsejaba a Walter Benjamin interpretar toda la obra de Kafka a partir del Libro de Job, donde, como es sabido, se cuenta la historia de Job, un hombre bueno, "perfecto y recto, temeroso de Dios y apartado del mal” (Job, I:I). Para poner a prueba su fe, le imponen desde arriba inmerecidas penalidades - ¿¿Será el hombre más justo que Dios?" (4:I7) -. Job, de igual modo que Josef K., el protagonista de El proceso, se pregunta qué mal ha cometido, se pregunta de qué es acusado. Pero el hombre no puede llevar razón frente a Dios, por mucho que aquél clame y se indigne contra el silencio de éste. No obstante, hay una diferencia esencial entre la historia del señor K. y la de Job, y ésta reside en la perspectiva del lector. En el caso de Job, éste no puede explicarse por qué es sometido al sufrimiento, siendo como es un hombre pío y cumplidor; pero el lector sí cuenta con una explicación, conoce la razón de sus penalidades, las cosas no son lo que parecen: Yahveh y Satanás han cerrado una apuesta en el Cielo para probar la fuerza de la fe de Job, la resistencia de sus convicciones. En el fondo, la historia para el lector tiene un sentido: él conoce la trampa. Aquí la parábola conlleva una enseñanza: sufre y sé paciente porque te espera el consuelo y la dicha. El lector no se siente solo.

En contrapartida, aunque el señor K. también trata de inquirir sobre el origen de su acusación, el lector está tan perdido como él. No hay explicación oculta. O al menos no se conoce. De ahí la profundad sensación de soledad que embarga al lector de esta historia. Nada tiene sentido, y Kafka no le otorga ni una tregua en sus pesquisas. Porque en el fondo el protagonista y el lector, y aún el propio autor, podrían identificarse, ser la misma persona. Y al no cerrar Kafka la historia, se abre la sospecha de la posibilidad de que todo pueda carecer de sentido: hemos leído una triste parábola sin enseñanza. ¿Era de verdad todo un sinsentido?

Y ciertamente no otro es el argumento de la novela de Kafka: el señor K. es sometido de improviso a un proceso sin que nadie sepa explicarle los motivos. Quizá su única culpa sea el mero hecho de haber nacido: mellius illi era si natus non fuisset. Con lo cual, el sentido del texto se amplifica a consideraciones teológicas. Y en miniatura, esta misma situación se reproduce de nuevo en el relato o parábola Ante la ley: un campesino pretende penetrar en la Ley, atravesar sus puertas, y, ante la prohibición del guardián de la entrada, consume su vida entera esperando allí, ante la puerta abierta, sin (poder) entrar. 


\section{3. "El mal" como punto de partida}

En el punto segundo hemos puesto de manifiesto el sobresaliente parecido entre la estructura significativa del relato kafkiano y la historia bíblica de Job. Las resonancias bíblicas del texto pues no solo están inducidas por el estudio de las circunstancias biográficas de su autor, sino también por la naturaleza de los propios interrogantes allí desplegados y la forma de plantearlos: la ausencia de respuesta a una pregunta que trasciende los detalles particulares o la existencia de un mal sufrido por un inocente. En este punto examinaremos nuevas resonancias bíblicas desde el punto de vista del pecado original: ¿qué mal ha cometido Josef K.?

La primera frase de Elproceso condensa la esencia de toda la historia, como muy agudamente señala Schirrmacher (2008). Así ocurre también en otros de sus relatos, como por ejemplo en La metamorfosis o en Un informe para una academia, donde toda la situación queda definida en unas pocas palabras iniciales. La primera frase de $E l$ proceso, vio la luz el ıo de agosto del año I9I4 (Stach, 20IO: 537). Kafka arranca con un nuevo proyecto: "Alguien tuvo que haber calumniado a Josef K. (Jemand musste Josef K. verläumdet haben), pues sin que hubiera hecho nada malo (denn ohne dass er etwas Böses getan hätte), una mañana fue arrestado (wurde er eines Morgens gefangen)" .

Llama la atención el uso del verbo verläumden, "calumniar" ("alguien tuvo que haber calumniado a Josef K.”). Este verbo alemán aparece definido en el diccionario de los hermanos Grimm (I852) como: "manchar la reputación de alguien a través de un falso testimonio". La RAE lo define como "imputar falsamente un delito". La nota común es la mendacidad del que imputa. Por tanto, el narrador, así como el lector, presumen la inocencia del protagonista de la novela, sin que por otra parte exista prueba fehaciente, pues la forma verbal "tuvo que" entraña un matiz hipotético. Lo primero que se nos comunica pues es un hecho verosímil, algo que quizá alguien había comentado. La intención del escritor desde el principio es no dejar nada claro. Y esa es la característica de toda la obra. ¿Estaba Kafka convencido de la inocencia de K.? En cualquier caso, la apunta con un margen de error, un margen que puede cambiar por completo el sentido de la historia.

Esta frase fue reformada al día siguiente, tal y como se observa en el manuscrito. La primitiva palabra gefangen (apresado) es tachada y sustituida por la que ha llegado a nosotros, verhaftet (arrestado). En opinión de Stach (2010: 537), la razón de tal cambio, aparte de la connatural inseguridad de su autor y su extremo perfeccionismo -“mis dudas se levantan alrededor de cada palabra" (2006: IO3)-, estriba en que esa palabra destilaba ciertas resonancias bélicas. Teniendo en cuenta que en ese mismo año comenzó la Primera Guerra Mundial, el primer término, gefangen, sonaba demasiado a un contexto militar. "Arrestar" induce a pensar en un contexto más civil y cotidiano.

Desde este momento, se inicia un peculiar proceso contra él, del que el acusado no sabe nada. Pareciera este planteamiento una astucia del escritor para captar la atención del lector, quien, consciente de la inocencia del protagonista, del tal señor K., lo acompañará 
en su inverosímil calvario, como el intrépido Cary Grant sufrió el suyo en la película de Hitchcock Con la muerte en los talones (1959), perseguido por unos malhechores que lo toman por otra persona. La primera frase presenta además un binomio irreductible heredado de la tradición religiosa: inocencia/maldad. K. no ha hecho nada malo (etwas Böses). ¿Qué es este "algo malo"? ¿Existe algo así, un mal en el que el hombre podría caer, que se le ofrece como posibilidad, a un hombre quien, viéndose sometido por tantas cadenas, curiosamente sí que dispone de libertad para la maldad? En el relato de Job, los males tienen su origen en una apuesta entre Jahveh y Satanás, pero aquí no se contempla ningún origen. La novela con todo, a juicio de Schirrmacher (2008), emula el inicio bíblico del Génesis. El hombre, gozando del regalo divino de la inocencia primordial, ha debido cometer ("tuvo que haber" cometido...) sin embargo un pecado y debe purgar por ello. En la dogmática cristina se propone al respecto la metáfora de Adán y Eva. Nuestros primeros padres cometieron el pecado original y nosotros, a fuer de descendientes, estamos ya para siempre manchados, expulsados del Paraíso. Cristo, encarnándose en el hombre y muriendo por él, vendría a redimirnos del pecado, pero esto formaría ya parte del Nuevo Testamento.

El hecho señalado por Kafka puede aludir sin embargo tanto a una anécdota posible y casi irrisoria (alguien es acusado por error), como a una grave concepción humana de inspiración teológica, definida por la mancha original del pecado, a la que el hombre no puede sustraerse. En un movimiento hermenéutico circular y sugerente, Schirrmacher relaciona "el mal” del comienzo de la novela de Kafka con la "vergüenza" a la que se alude en el cierre del libro. Vergüenza es lo que sintieron Adán y Eva tras cometer el pecado original. Se vieron entonces por primera vez desnudos. "A Josef K. lo que le sobrevive es la vergüenza, no la fama ni la gloria”, señala Córdoba Ospina (2015: 6), quien llama la atención de cómo la problemática relación de la literatura con la realidad se juega en el carácter metafórico de la primera, contra el que habría luchado Kafka, porque "la literatura debe ser un acontecimiento en sí mismo". Adorno, por el contrario, subraya la literalidad de la escritura kafkiana (I962: 264), pero de la misma manera que se puede interpretar literalmente la Torá o el Antiguo Testamento. Es decir, subsiste en las palabras sagradas una tensión irrebasable entre el contenido de las mismas, que inevitablemente tienen una referencia, y el sentido hacia el que apuntan, violentando la finitud de su contenido semántico.

Quizá estas referencias a la vergüenza y hacia el mal fuerzan en exceso la lógica del texto, pues, como vimos en el primer apartado, en realidad el cierre de la obra estuvo a cargo de Brod. Pero no parece impertinente el sentido apuntado por Schirrmacher. La Biblia no sería en el fondo sino la historia de un gigantesco proceso contra la humanidad, que se ha alejado de su Creador. Si al principio fue el Verbo, si el efecto hipnótico de la oración, que consiste en la repetición sin fin de unas palabras escogidas, se identifica con el de la religión, de modo que la religión ha sido en el fondo literatura, ¿habrá querido Kafka seguir el proceso contrario?, ¿habrá querido convertir la literatura en religión? Tal condensación de significados, de excedentes semánticos, sólo podía dar 
lugar a una obra fragmentaria, aforística, incompleta, agotada en el primer arranque debido a la sobreabundancia de riqueza semántica, donde no se tienen tanto en cuenta las grandes líneas de tensión dramática, sino la riqueza orfebre del detalle, cual especie de microcosmos que no se subordina a grandes líneas de arquitectónica musical sino que las refleja en miniatura. De esta lógica "micro-macro" nos ocuparemos en el siguiente apartado.

Demasiado peso pues recae sobre los hombros de un vulgar trabajador de banco, simbolizado humildemente por la letra K., que no sabe explicarse a la sazón por qué se halla inmerso en un proceso. Todo transcurre de forma opaca e inaccesible a su conocimiento y a su voluntad, y aunque intenta saber, nadie le sabe decir. Por eso la parábola Ante la ley es esencial en la novela, porque por fin se presenta alguien, el capellán de la cárcel, que no solo forma parte de las autoridades que han de juzgarlo, es decir, es alguien del "otro lado", sino que además está dispuesto a explicarle su situación. Algunos estudiosos han considerado esta parábola incluso como una llave de acceso para toda la obra de Kafka: Brod (I959: I9) o Henel (I963: 69). De modo que este pasaje representa el punto en el que más cerca estamos (como lectores, que también andamos perdidos) de una explicación oficial de lo que ocurre con Josef K. (Purdy, r968: 4IO).

\section{Un núcleo de inspiración}

La parábola ostenta de forma condensada, según ha mostrado Uwe-Jürgen Ness (2013) la estructura básica de sus novelas más célebres. Ante la ley actúa como una especie de reproducción fractalizada respecto a la novela completa. O como dice Rubio (20IO: 137), es una mise en abime, porque un relato dentro de otro reproduce en menor formato la experiencia del protagonista textual y metatextualmente. Kafka habría tomado como núcleo inspirador de su novela la parábola, esta es la hipótesis. Porque, por un lado, la trama imprevisible de sinsentido se reproduce paralelamente en los dos niveles, y por otro, la propia confección de numerosos detalles es casi idéntica.

A Josef K., casi al final de la novela, le han encargado en la oficina que acompañe a un cliente italiano en una visita turística por la catedral. El escenario no es neutral, porque las proporciones del recinto sagrado le parecen a Josef K. ir más allá de lo que el hombre puede soportar. El cliente italiano no aparece, y es allí dentro de la catedral donde Josef K. se ve abordado por el religioso. Éste le advierte de que se está engañando sobre su propio proceso, y entonces le cuenta la parábola. ¿No era acaso la parábola el medio idóneo del que se servía Jesucristo para ilustrar a los fieles y hacerles comprender por medio de imágenes? Nos encontramos de nuevo con resonancias bíblicas.

El capellán le advierte de que el juicio va mal, de que “acabará mal. Te consideran culpable" (Kafka, I998: 2I8). Por un instante, se crea la ilusión de que realmente el juicio se desenvuelve siguiendo alguna lógica, cosa que hasta ahora no ocurría, pues ninguna palabra se podía tomar en sentido literal (Stach, 20IO: 548): ¿qué tipo de interrogatorio es 
el que llevan a cabo los vigilantes del inicio que se comportan y van vestidos de manera tan peculiar?, ¿en qué consiste un arresto que no tiene ninguna consecuencia práctica en su vida y que se agota en su propia notificación? Los momentos concretos están presentados de forma realista, pero la conexión entre ellos no están regidos por la causalidad, igual que ocurre en los sueños, donde una escena puede desembocar en cualquier otra. Es un cuadro imprevisible. Su lógica es onírica con tintes expresionistas. Pero al llegar a este momento de la narración, parece fulgurar un hilo de coherencia, por fin alguien se digna a una aclaración. Pero el viraje de Kafka, una vez más, le lleva a explicar una situación enigmática (la situación de K.) a través de otra no menos enigmática (la del campesino).

Respecto a la confección de los detalles entre la macro- y la micro-narración, los paralelismos saltan a la vista. El guardián deja la puerta en franquía, situándose en un lateral, sin obstaculizar su acceso. Le advierte al campesino de que por ahora (jetzt) no puede entrar y que él es poderoso, es más, una serie de guardianes más poderosos que él le esperarían en las sucesivas puertas. Los que detienen a Josef K. también se confiesan como "niedrige Angestellte", como los últimos de una cadena, por consiguiente ellos en realidad no son la principal amenaza. En ambos casos se desplaza la responsabilidad a instancias superiores. La culpa no reposa en ninguna cabeza visible. Todos siguen obediente y asépticamente el procedimiento sin apelar a la conciencia propia. Este aspecto le ha llevado a Hannah Arendt (2005: 92) a enfocar la situación desde una óptica sociológica. K. está a merced de una jerarquía corrupta y mentirosa que no se siente responsable, solamente obligada. De hecho, el abogado de K., como luego más tarde el capellán en la catedral, le aconseja que lo mejor que puede hacer es adaptarse al sistema, en lugar de luchar contra él. Aparentemente todo funciona como debe, de suerte que responde idóneamente a las necesidades de aquellas personas que temen la libertad. Si el mecanismo funciona necesariamente así, nada hay que hacer por cambiarlo. Lo contingente ha devenido necesario, y de este modo se ha legitimado. Y un ocasional desperfecto corre a cuenta del infractor, no del sistema, infractor que desarrolla fácilmente un sentimiento de culpa. Arendt está convencida de que la crítica contra la burocracia excesiva, que aniquila a los propios actores sociales, es lo que mejor caracteriza el mensaje de esta novela. Piensa que otras interpretaciones, como la psicoanalítica (la inhibición frente a la autoridad del padre), o la religiosa (la Ley como la representación de un dios distante), aunque “aparentemente más profundas" (94), son erróneas. Esta pensadora sostiene que la intención de Kafka sería precisamente la contraria: criticar una situación que se ha tomado por natural sin serlo, y Kafka, mostrando al mundo tal cual es, injusto y cruel, lo despoja de su apariencia de fatalidad, en el sentido de designio divino (después de todo, el que cuenta la parábola de Ante la ley es un sacerdote).

Frente a Arendt, pienso que, de entrada, otras posibles interpretaciones no tienen por qué ser "erróneas", pueden ser incluso complementarias, aunque unas resultan más fructíferas que otras. Kafka dejó obviamente el sentido de sus textos abierto, y no podemos cerrarlos con una hermenéutica definitiva. De hecho, dado el carácter introspectivo y retraído de su autor, posiblemente estuviera manteniendo más una lucha intestina 
consigo mismo y con su destino, tal y como traslucen sus diarios: "puedo entablar de nuevo un diálogo conmigo mismo [...] sólo por esta vía puede haber una mejora para mí" (1995: 265). La verdadera revolución ha de llevarse en el ámbito más íntimo, a saber: el de la conciencia. Es su relación con el mundo lo que está en juego. No obstante la labor de análisis actúa como una linterna, iluminar una parcela, implica necesariamente dejar otras en la sombra; y la labor de la comprensión "es una tarea a la que nunca se puede dar cumplimiento por entero" (Gadamer, I977: 372). De modo que, siendo legítimo y aún certero el enfoque de Arendt, me parece que otros enfoques hacen más justicia a la riqueza del texto.

Un segundo paralelismo entre el relato y la novela se cifra en la duda que tiene el protagonista de que a fin de cuentas todo pudiera ser "falso". Josef K. se replantea el verdadero poder de los guardianes que van a arrestarlo: quizá se trate todo de una representación, de una inocentada de mal gusto, "todo podría ser visto como una broma (als Spass)" (г998: гі). En ese caso, lo único que tendría que hacer es reírse y seguir su propio camino. Pero ante la presión social, ante la virtual acusación de no ser capaz de entender una broma, se dice a sí mismo, actuemos (mitspielen) acorde. La resignación es asumida a causa del más nimio de los motivos. Y así ocurre también en la parábola: la resignación se acata al jobservar el aspecto del guardián! Éste lo anima a adentrarse en la Ley pese a su prohibición, el campesino no sabe qué hacer, duda, pero al observarlo decide inhibirse. El principal obstáculo para adentrarse parece ser él mismo. La espera ante la puerta durante toda una vida (independientemente de aquello que simbolice: ¿el padre, la verdad, el pecado, Dios?) parece un precio demasiado alto para tan escasa resistencia por parte del guardián. Quizá el campesino se considera indigno, tiene miedo o puede ser incluso que el guardián no exista y represente solamente la censura de su propia conciencia.

El campesino y Josef K. también comparten ofertas de soborno. En el primer caso, por iniciativa del campesino, ofertas que, por otro lado, el guardián de la puerta acepta, exclusivamente, aclara, para que el primero tenga la conciencia tranquila de haberlo intentado todo. En el segundo caso, son los guardianes quienes le piden a K. que les confíe sus pertenencias en depósito. Que los mismos encargados de perpetuar el sistema estén corrompidos, da idea de la perversión del sistema: aquellos que exigen fidelidad a las reglas son los primeros en violarlas. Cuando el capellán, en su intento de asesorarlo, le explica que las palabras no han de ser examinadas necesariamente bajo el prisma de su valor de verdad, sino del de su necesidad (Notwendigkeit), no hace más que confirmar la poca consistencia que tiene el conjunto de la situación. De esas palabras K. deduce que "la mentira se ha convertido en el orden del mundo" (ı998: 270).

¿Y qué relación mantiene el acusado con semejantes operarios de la ley? Los diálogos son rutinarios, impersonales, los guardianes ni saben ni quieren saber. La implicación es nula. Es decir, los mediadores no aportan nada significativo al estado del reo. K. y el campesino por su parte ofrecen nula resistencia frente a ellos, aceptan la situación como si fuera inevitable, no hay un conato de rebelión, pese a la ausencia 
de intimidación física o de otra cualquier clase y a que sus guardianes no se encuentran oficialmente identificados de ninguna manera. En consecuencia, ya desde el inicio se encarrilla un proceso de opacidad: el "proceso" es tan inasequible para K. como "la Ley" para el campesino, y los intentos por saber más son tan infructuosos como desencaminados.

\section{El ( $\sin )$ sentido del sufrimiento}

¿Tiene sentido la larga espera del campesino, que no desemboca en nada, más que en la muerte? ¿Qué hay más allá de las puertas de la Ley, si es que hay algo? ¿Tienen sentido las inquisiciones de Joseph K.? La Ley por definición establece un orden de acceso público de correspondencia entre una acción y sus consecuencias. ¿Qué clase de ley puede autofundamentarse en su propia opacidad? Cuando el guardián se percata de que el campesino está próximo a su muerte, le revela un hecho enigmático: "esta puerta (dieser Eingang) era accesible solo para ti (war nur für dich bestimmt)" (I998: 263). Y en ese momento cierra la puerta y se marcha. De esta forma se resuelve la promesa de explicaciones que la parábola anunciaba en medio del desconcierto de toda la novela. ¿Una muerte absurda? El centro de gravedad, y esto lo ha anotado muy bien Cabrera (2009), ya no pivota sobre el problemático final de la historia (si el campesino tendría que haber atravesado la puerta, si Job estaba legitimado a perder su fe en un dios apostador), sino sobre el propio camino de la vida: ¿el sufrimiento acumulado está justificado siquiera por un final feliz? El sufrimiento personal se subsume en una lógica total que escapa al actor individual, quien ya ha perdido la esperanza: "lo que resuena en Kafka no es un mensaje, sino sus ruinas" (Mate y Mayorga 2000: 59).

La Ley no es accesible porque quizá su misterio, su oscuridad, constituyan su mayor protección, su más perfecta legitimación. Josef K. es un personaje auténtico que vive en un mundo falso, y así lo deduce él mismo casi al final del capítulo (y del libro): "la mentira (die Lüge) se ha convertido en el orden del mundo (wird zur Weltordnung gemacht)" (1998: 270). Pero pienso que esta falsedad o indigencia de sentido no se refiere a este o aquel aspecto parcial del mundo. Precisamente el vértigo que provocan los escritos de Kafka se debe a que es el mundo en su conjunto, y no una parte de éste, el que no parece consolar ante la voz que se alza preguntando un porqué.

El relato bíblico de Job se resuelve con la aceptación de la propia ignorancia -“yo hablaba lo que no entendía" (42: 3) - y con el arrepentimiento de Job: "mas ahora mis ojos te ven. Por tanto me aborrezco, y me arrepiento en polvo y ceniza" $(42: 5,6)$. Pero en el camino ha quedado una reivindicación de la autonomía moral: ¿quién se equivoca? Y Job se arrepiente, sin que Yahveh le explique nada sobre su apuesta con Satanás. Sin mediar excusas. No da ninguna explicación, solamente ostenta su omnipotencia, ante la que Job se maravilla y cede. El Dios bíblico es un Dios que se esconde también bajo su inaccesibilidad. Se sitúa, como lo guardianes, más allá de su propia ley. 
Esta línea de argumentación puede conducir a consecuencias demasiados escabrosas en el terreno de la teología o de la metafísica. Otros intérpretes como Mate y Mayorga (2OOO) han preferido leer El proceso en clave antropológica, "una novela sobre el desamparo en que cada hombre es dejado por los demás” (2000: 60). Estos autores se apoyan en W. Benjamin (2000: 59) para trazar la genealogía de este asilamiento. El mundo moderno ha perdido su encantamiento, su aura, y los hombres, aislados entre sí, solo pueden sentir ante el otro miedo o indiferencia. Por tanto la denuncia de Kafka sería la de la soledad o la nuda incomunicación.

Del mismo modo que en el caso de Arendt, queplanteaba unaclave interpretativa en términos de crítica socio-política, pienso que esta clave antropológica de Mate y Mayorga, en términos de deshumanización, como resultado del proceso de la modernidad, no hace justicia a la profundidad de las alegorías kafkianas Sostengo que más que a Benjamin, hay que seguir el consejo de Scholem (I975: 2I2). Mi hipótesis es que, aún superando las contradicciones sociales, o multiplicando las vías comunicativas entre los hombres, para Kafka no se habría solucionado el problema. Porque la angustia no es específica ante un estímulo determinado, sino ante una forma de ser el mundo. La dureza enigmática con la que cierra sus historias, y en especial Ante la ley, parecen clamar contra el mismo silencio del universo.

\section{Conclusiones}

He intentado mostrar cómo la parábola Ante la ley condensa en cierto modo el sentido de la escritura para Kafka. El hecho de publicarla dos veces e incluirla en su novela Elproceso, como síntesis aclaratoria de toda la historia, da idea de su trascendencia y del valor que debió atribuirle el autor. Además, en la novela tiene una doble función: como clave aclaratoria del proceso, tanto para el lector como para el protagonista, aunque al fin y al cabo su interpretación acabe siendo tan enigmática y abierta como la del conjunto de la novela; y como representación en miniatura del todo en el que se inscribe: no sólo por los numerosos paralelismos narrativos que surgen entre la parte y el todo, sino también por las cuestiones trascendentales hacia las que apuntan ambos niveles.

¿Cuáles son esas cuestiones? Esta parábola surge en un momento biográfico en el que Kafka se interesa intensamente por las doctrinas judías, con lo que se abona el terreno para una posible interpretación de cuño teológico (Schirrmacher) o más metafísico (Scholem), y no tanto político (Arendt) o social (Mate y Mayorga), siendo conscientes por supuesto del carácter estructuralmente abierto de los escritos kafkianos, tanto desde el punto de vista de su gestación (como en el caso que nos ocupa), como el de su interpretación.

Aunque en Kafka no aparezca como un tema explícito, pienso que su sensibilidad religiosa funciona como el negativo de una fotografía: no hay evidencia tácita alguna, no se tematiza expresamente, pero no por ello deja de latir y presionar la pregunta 
acerca del sentido en términos muy radicales. De hecho, hemos comprobado a través de dos ejemplos del Antiguo Testamento -la historia de Job y la del pecado original en el Génesis-, con qué facilidad se pueden superponer estructuralmente dichas narraciones.

Y no puede aparecer explícitamente porque Kafka es el maestro de la elusión. El proceso comienza con una presunción, la de virtual inocencia de su protagonista y termina con una ambivalencia: propone a un mismo tiempo la ausencia de esperanza y la renuncia a desesperar. El lector tendrá que optar. 


\section{Referencias bibliográficas}

-Adorno, Theodor W. (ig62). "Apuntes sobre Kafka”, en: Prismas. La crítica de la cultura y la sociedad(tr. Manuel Sacristán). (26o-292), Barcelona; Ariel.

- Arendt, H. (2005). Ensayos de comprensión: 1930-1954; escritos no reunidos e inéditos de Hannah Arendt. Madrid: Caparros editores.

- Brod, M. (1959). Verzweiflung und Erlösung im Werk Franz Kafkas. Frankfurt/M.: Fischer.

- Cabrera, I. (2009). Kafka a través de Job, Job a través de Kafka. Tópicos del seminario, 22, I57-I74.

- Córdoba Ospina, G. (20I5). La "metáfora" como dadora de muerte. Acerca de la última frase de El Proceso de Kafka. Alabe, II.

- De Jerusalem, E. B. (i994). Biblia de Jerusalén. Madrid: Alianza Editorial.

- Gadamer, H. G. (i977). Verdady método. Salamanca: Sígueme.

- Grimm, J., \& Grimm, W. (1852). Das deutsche Wörterbuch. Hamburg: Hoffmann und Campe.

- Grözinger, K. E. (20I4). Kafka und die Kabbala: Das Jüdische im Werk und Denken von Franz Kafka. Frankfurt/M.: Campus Verlag.

- Henel, I. (1963). Die Türhüterlegende und ihre Bedeutung für Kafkas 'Prozeß'. Deutsche Vierteljahrsschrift für Literaturwissenschaft und Geistesgeschichte, 37(I), 50-7O.

- Kafka, F. (1995). Diarios (1910-1923). Barcelona: Lumen-Tusquets.

- Kafka, F. (1998). Der Prozess. Berlin: Dtv.

- Kafka, F. (1998). Carta a mi padre y otros escritos. Buenos Aires: Emecé.

- Kafka, F. (2006). Tagebücher/1: 19o9-1912; in der Fassung der Handschrift. Frankfurt/M.: Fischer.

- Löwy, M. (2019). Redención y Utopía: Eljudaísmo libertario en Europa central. Un estudio de afinidad electiva. Santiago de Chile: Ariadna Ediciones.

- Mate, R., y Mayorga, J. (200o). “Los avisadores del fuego”: Rosenzweig, Benjamin, Kafka. Isegoría, $23,45^{-67}$. 
- Purdy, S. B. (ig68). A Talmudic Analogy to Kafka’s Parable "Vor Dem Gesetz". Papers on language and literature, $4(4), 420$

- Rubio, F. B. (20Iо). "Die Lüge wird zur Weltordnung gemacht": procesos victimarios y distorsión de la objetividad en la obra de Franz Kafka. Revista de Filología Alemana, I8, I35I6I

- Schirrmacher, F. (2008, 3 de julio). Neunzehn Worte Kafka. FAZ. Obtenido el o2 de marzo desde https://www.faz.net/aktuell/feuilleton/buecher/kafkas-saetze-I-neunzehnworte-kafka-I66 57 I8.html

- Scholem, G. (1975). Walter Benjamin: die Geschichte e. Freundschaft. Frankfurt/M.: Suhrkamp.

- Stach, R. (20IO). Kafka: die Jahre der Entscheidungen. Frankfurt/M.: Fischer.

- Ness, U. (20IO). Integration in die Welt des Schlosses?-Die Türhüter-Legende Franz Kafkas im Kontext der beiden Romane Das Schloß und Der Prozeß. Obtenido el I5 de febrero desde http://uweness.eu/das-gesetz-kafkas.html 